\title{
Ant-based Clustering Algorithms: A Brief Survey
}

\author{
O.A. Mohamed Jafar and R. Sivakumar
}

\begin{abstract}
Ant-based clustering is a biologically inspired data clustering technique. Clustering task aims at the unsupervised classification of patterns in different groups. Clustering problem has been approached from different disciplines during last years. In recent years, many algorithms have been developed for solving numerical and combinatorial optimization problems. Most promising among them are swarm intelligence algorithms. Clustering with swarm-based algorithms is emerging as an alternative to more conventional clustering techniques. These algorithms have recently been shown to produce good results in a wide variety of real-world applications. During the last five years, research on and with the ant-based clustering algorithms has reached a very promising state. In this paper, a brief survey on ant-based clustering algorithms is described. We also present some applications of ant-based clustering algorithms.
\end{abstract}

Index Terms-Ant-based Clustering, Data Mining, Cluster Analysis, Swarm Intelligence

\section{INTRODUCTION}

With substantial decline in data storage cost, rapid advancement in computer networks, technology advances in data acquisition, improvement in computer performance and explosive growth in the generation of electronic information, huge amount of data are being collected and stored in databases. The amount of data stored in databases continues to grow fast. This large amount of stored data contains valuable hidden knowledge, which could be used to improve the decision-making process of an organization. It is therefore no simple matter to discover business intelligence that must assist decision making. Hence, such large databases have led to the emergence of a field called data mining and knowledge discovery in databases [32]. Data Mining refers to extracting or mining knowledge from large amounts of data. It involves the use of data analysis techniques to discover previously unknown, valid patterns and relationships in large data sets. This encompasses a number of technical approaches such as clustering, data summarization, classification, finding dependency networks,

Manuscript received May 22, 2010.

O.A. Mohamed Jafar is with the Department of Computer Science, Jamal Mohamed College (Autonomous), Tiruchirappalli, Tamil Nadu, India and doing research in Computer Science, A.V.V.M. Sri Pushpam College (Autonomous), Poondi, Thanjavur, Tamil Nadu, India under Bharathidasan University, Tiruchirappalli. Phone: 0431-2422333, 9865080012; (e-mail:mohamedjafaroa@yahoo.in).

R. Sivakumar is with Department of Computer Science, A.V.V.M. Sri Pushpam College (Autonomous), Poondi, Thanjavur, Tamil Nadu, India. Phone: 9443662536; (e-mail: rskumar.avvmspc@gmail.com). regression, analyzing changes and detecting anomalies.

Ants belong to social category. It means that the ant would be unable to survive on its own. But colonies of ants can exhibit remarkable coordination of activities between the individuals. This coordination does not stem from a 'center of control' rather it is self-organizing. When ants search for food, they initially perform a random walk search in their local neighborhood. As the ants move, they deposit a chemical substance called pheromone on their path. This substance is perceived by other ants, helping them to make decision on their next move. This way a solution is constructed in an incremental fashion. The amount of pheromone laid is proportional to the number of ants that used in the route. Some examples are building an ant nest, brood pits and cemeteries, hunting and foraging food [16] [10].

The communication between the agents during the search process is not direct, instead they communicate indirectly by modifying the environment faced by each other. There is no single 'Ant Model', rather there exists a family of models, each inspired by a different aspect of ant behavior. These models include those inspired by : i. Ant-foraging behavior ii. Brood-sorting behavior iii. Cemetery formation behavior and iv. Cooperative transport. Ant colonies show high degrees of parallelism, self-organization and fault tolerance. These characteristics are essential for the computer systems.

Clustering means the act of partitioning an unlabeled dataset into groups of similar objects. The goal of clustering is to group sets of objects into classes such that similar objects are placed in the same cluster while dissimilar objects are in separate clusters. Clustering is used as a data processing technique in many different areas, including artificial intelligence, bioinformatics, biology, computer vision, city planning, data mining, data compression, earth quake studies, image analysis, image segmentation, information retrieval, machine learning, marketing, medicine, object recognition, pattern recognition, spatial database analysis, statistics and web mining [39].

The nature inspired methods like ant-based clustering techniques have found success in solving clustering problems. They have received special attention from the research community over the recent years. It is because these methods are particularly suitable to perform exploratory data analysis, and also because there is still a lot of investigation to perform on this field - the research nowadays concentrates on improving performance, stability, convergence, speed, robustness and other key features that would allow us to apply these methods in real applications. The main research on the nature inspired methods does not focus on the strict 
modeling of the natural processes; it merely focuses on using the best ideas to improve the convergence and accuracy of such methods.

The remainder of this paper is structured as follows. In section 2, swarm intelligence concept is described. In section 3 , an overview of ant colony system is presented. Section 4 contains cluster analysis. Section 5 reviews the previous work on ant-based clustering. Furthermore, the applications of ant-based clustering algorithms are presented in section 6 . Finally, conclusion and future research directions are presented in section 7 .

\section{SWARM INTELLIGENCE}

Swarm Intelligence (SI) [42] [8] [1] is an innovative, distributed intelligent paradigm for solving optimization problems that organized from the study of colonies, or swarm of social organisms. Studies of the social behavior of organisms (individuals) in swarm prompted the design of very efficient optimization and clustering algorithms. The state of the art clustering algorithms based on SI tools are Particle Swarm Optimization (PSO) and Ant Colony Optimization (ACO).

PSO is a new evolutionary computation technique first introduced by Kennedy and Eberhart in 1995 [43]. It is a stochastic optimization approach, modeled on the social behavior of animals such as a flock of birds, a school of fish, or a swarm of bees or a group of people who pursue a common goal in their lives [42]. PSO is a population-based search procedure where the individuals, referred to as particles, are grouped into a swarm. Each particle in the swarm represents a candidate solution to the optimization problem. In a PSO system, each particle is "flown" through the multidimensional search space, adjusting its position in search space according to its own experience and that of neighboring particles. A particle therefore makes use of the best position encountered by itself and the best position of its neighbors to position itself toward an optimal solution.

The effect is that particles "fly" toward an optimum, while still searching a wide area around the current best solution. The performance of each particle (i.e. the "closeness" of a particle to the global minimum) is measured according to a predefined fitness function which is related to the problem being solved.

The main strength of PSO is its fast convergence, which compares favorably with many global optimization algorithms like Genetic Algorithms (GA), Simulated Annealing (SA) and others. For applying PSO successfully, one of the key issues is finding how to map the problem solution into the PSO particle, which directly affects its feasibility and performance. Applications of PSO include function approximation, clustering, optimization of mechanical structures, and solving systems of equations.

ACO approach was proposed in 1992 by Marco Dorigo et al. to solve several discrete optimization problems [7] [18] [22]. ACO deals with artificial systems that is inspired from the foraging behavior of real ants, which are used to solve discrete optimization problems [20]. The main idea is the indirect communication between the ants by means of chemical pheromone trials, which enables them to find short paths between their nest and food.

Studies of ant colonies have contributed in abundance to the set of intelligent algorithms. The modeling of pheromone depositing by ants in their search for the shortest paths to food sources resulted in the development of shortest path optimization algorithms.

\section{ANT COLONy SySTEM}

The basic idea of real ant system is illustrated in Figure 1. The ants move in a straight line from nest to food source (Figure 1 (a)). At the next stage, assume that there is an obstacle (Figure 1 (b)). In this case, to avoid the obstacle initially each ant chooses to left or right at random (Figure 1 (c)). Let us assume that ants move at the same speed depositing pheromone in the trail uniformly. However, the ants that, by chance, select to turn left will reach the food source sooner, whereas the ants that turn right will follow a longer path. The intensity of pheromone deposited on shorter path is more than the other path. So ants will we increasingly guided to move on the shorter path (Figure 1 (d)). The intensity of deposited pheromone is one of the most important factors for ants to find the shortest path.

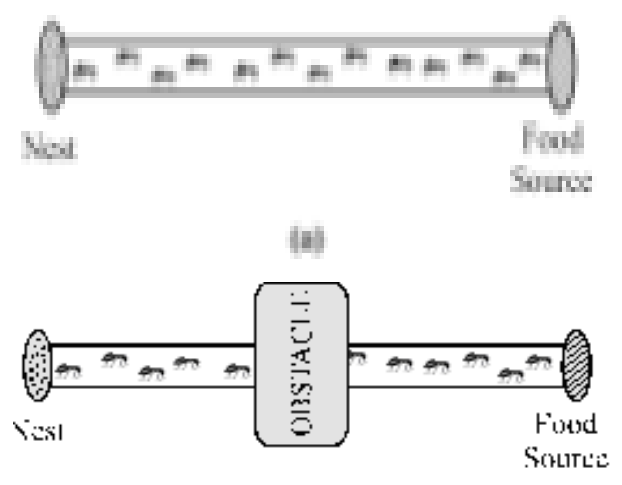

(b)
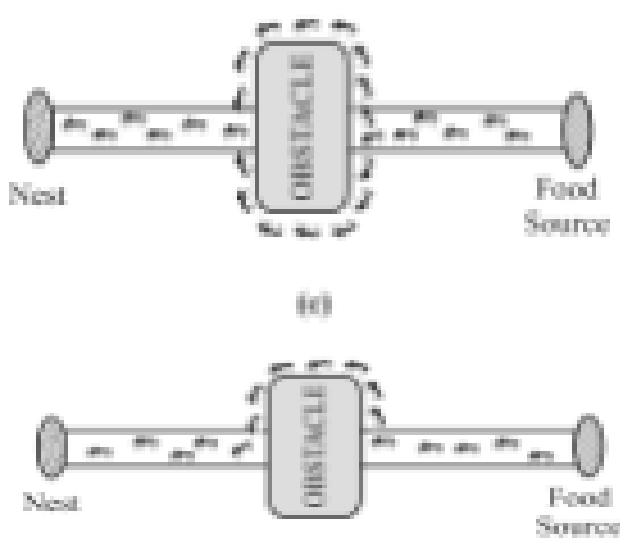

(iil)

Figure 1. Illustrating the behavior of real ant movements

1) Ants following a path between their nest and food source

2) Encountering obstacle of ants

3) Selecting of ants

4) Finding shortest path of ants

The ACO is a meta-heuristic inspired by the behavior of some species of ants that are able to find the shortest path 
from nest to food sources in a short time. The meta-heuristic was introduced by Margo Dorigo [21] and his colleagues [8] in the early 1990's.

The ACO mimics the way real ants find the shortest route between a food source and their nest. The ants communicate with one another by means of pheromone trails. Pheromone is the chemical material deposited by the ants, which serves as critical communication medium among ants, thereby guiding the determination of the next movement. The more the number of ants traces the given path, the more attractive this path (trail) becomes and is followed by other ants by depositing their own pheromone. This auto catalytic and collective behavior results in the establishment of the shortest route. Ants find the shortest path based on intensity of pheromone deposited on different paths. This characteristic of ants is adapted on ant colony optimization algorithms to solve real problems.

Relevant ACO techniques have been successfully employed in many fields, such as traveling salesman problem, scheduling, vehicle routing, quadratic assignment problem, routing optimization in telecommunication networks and graph coloring.

\section{CluSTER ANAlysis}

The clustering problem is the ordering of a set of data into groups, based on one or more features of the data. Cluster analysis [15] [39] [44] is an unsupervised learning method that constitutes a main role of an intelligent data analysis process. It is used for the exploration of inter-relationships among a collection of patterns, by organizing them into homogenous clusters. It is called unsupervised learning because unlike classification (known as supervised learning), no a priori labeling of some patterns is available to use in categorizing others and inferring the cluster structure of the whole data.

Cluster analysis is a tool for exploring the structure of data. Clustering is the process of grouping objects into clusters such that the objects from the same clusters are similar and objects from different clusters are dissimilar. The relationship is often expressed as similarity or dissimilarity measurement and is calculated through distance function. Clustering is useful technique for the discovery of data distribution and patterns in the underlying data. It is an unsupervised learning technique. Unsupervised learning is learning from observations and discovery. In this mode of learning, there is no training set or prior knowledge of the classes. The system analyses the given set of data to observe similarities emerging out of the subsets of the data. The outcome is a set of class descriptions, one for each class, discovered in the environment. The basic objective of clustering is to discover both the dense and sparse regions in a data set. In cluster analysis, one does not know what classes or clusters exists and the problem to be solved is to group the given data into meaningful clusters.

The aim of clustering analysis is to find any interesting groupings of the data. It is possible to define cluster analysis as an optimization problem in which a given function consisting of within cluster similarity and between clusters dissimilarities needs to be optimized.
A problem with the clustering methods is that the interpretation of the clusters may be difficult. In addition, the algorithms will always assign the data to clusters even if there were no clusters in the data. Therefore, if the goal is to make inferences about its cluster structure, it is essential to analyze whether the data set exhibits a clustering tendency. In real-world application, there may be errors (called noise) in the collected data set due to inaccurate measurement or due to missing values. Therefore a pre-processing step is needed.

Cluster analysis is a difficult problem because many factors (such as effective similarity measures, criterion functions, algorithms and initial conditions) come into play in devising a well tuned clustering technique for a given clustering problem. Moreover, it is well known that no clustering method can adequately handle all sorts of cluster structures (shape, size and density). Sometimes the quality of the clusters that are found can be improved by pre-processing the data. Another common technique is to use post-processing steps to try to fix up the clusters that have been found.

Generally clustering algorithms can be categorized into hierarchical methods, partitioning methods, density-based methods, grid-based methods, and model-based methods [5].

The desirable features of clustering algorithms are scalability, ability to deal with different data types, discovery of clusters with arbitrary shape, able to deal with noise and outliers, insensitive to order of input records, incorporation of user-specified constraints, interpretability and usability, minimal requirements for domain knowledge to determine input parameters.

There exist a large number of clustering algorithms in the literature [75]. No single algorithms is suitable for all types of objects, nor all algorithms appropriate for all problems. Unfortunately, many of the traditional clustering algorithms share a number of drawbacks. Recently, algorithms inspired by nature used for clustering. It is claimed that ant-based clustering algorithms can overcome these drawbacks. These algorithms have advantages in many aspects, such as self-organization, flexibility, robustness, no need of prior information, and decentralization [6].

\section{ANT-BAsed Clustering In THe Literature}

Ant colonies provide a means to formulate some powerful nature-inspired heuristics for solving the clustering problems. Several clustering methods based on ant behavior have been proposed in the literature. This section provides a brief description of these methods.

In several species of ants, workers have been reported to form piles of corpses - cemeteries - to clean the nests. Cheretien and others [12] [8] have performed experiments with the ant Lasius Niger to study the organization of cemeteries. Other experiments on the ant Phaidole Pallidula are also reported in Deneubourg et al. [16]. Brood sorting is observed by Franks and Sendova-Franks [27] in the ant Leptothorax Unifasciatus. Workers of this species gather the larvae according to their size. Franks and Sendova-Franks [27] have intensively analyzed the distribution of brood within the brood cluster.

Ant-based clustering sorting was first introduced by 
Deneubourg et al. [16] to explain the above mentioned phenomena of corpse clustering and larval sorting in ants [8]. It is an instance of the broad category of ant algorithms [23].

Ant-based clustering algorithms are based upon the brood sorting behavior of ants. Larval sorting and corpse cleaning by ant was first modeled by Deneubourg et al. for accomplishing certain tasks in robotics. Their work was actually focused on clustering objects by using group of realworld robots. Their model is known as basic model (BM). This model can be described as follows: The data items are randomly scattered into a two-dimensional grid. Initially, each data object that represents a multi-dimensional pattern is randomly distributed over the $2 \mathrm{D}$ space. Each ant moves randomly around this grid picking and dropping the data items. The decision to pick up or drop an item is random but is influenced by the data items in the ant's immediate neighborhood. The probability of dropping an item is increased if ants are surrounded with similar data in the neighborhood. In contrast, the probability of picking an item is increased if a data item is surrounded by dissimilar data, or when there is no data in its neighborhood. In this way, clustering of the elements on the 2D grid is obtained. Real ants Messor Sancta build clusters starting from randomly located corpses is shown in the following Figure 2.
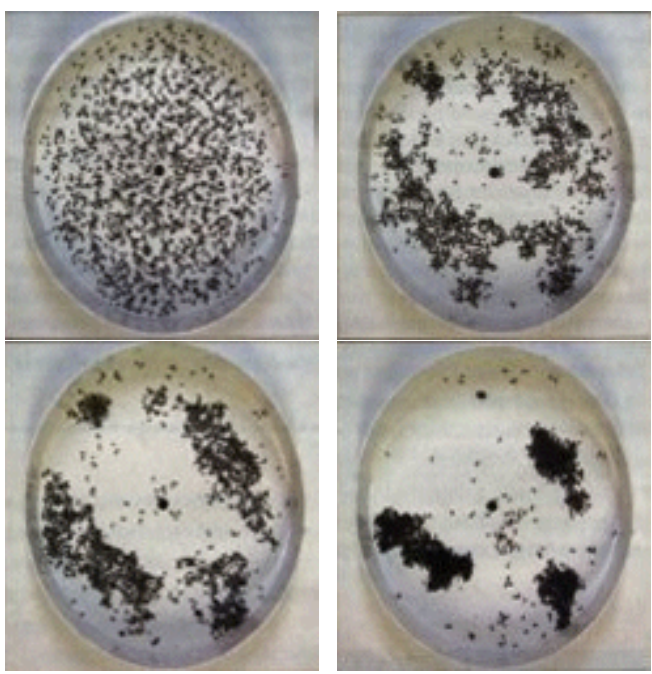

Figure 2. Real ant cluster the bodies of deal ants

The probability of picking an item is given by:

$$
P_{p}=\left(\frac{k_{1}}{k_{1}+f}\right)^{2}
$$

where

- $\mathrm{P}_{\mathrm{p}}$ is the probability of picking

- $\mathrm{f}$ is the perceived fraction of items in the neighborhood of the ant

- $\mathrm{k}_{1}$ - is a threshold item.

The probability of dropping an item is given by:

$$
P_{\mathrm{d}}=\left(\frac{\mathrm{f}}{k_{2}+f}\right)^{2}
$$

where:

- $\quad \mathrm{P}_{\mathrm{d}}$ is the probability of dropping

- $\mathrm{k}_{2}$ is another threshold constant.

Lumer and Faieta [50] modified Deneubourg et al.'s basic model [16] using a dissimilarity-based evaluation of the local density, in order to make it suitable for data clustering and it has subsequently been used in data mining [51]. This algorithm is called LF model or standard ant clustering algorithm (SACA). In this algorithm, each ant-like agent cannot communicate with each other, and they can only sense the similarity of the objects in their immediate region.

Lumer and Faieta [50] have introduced the notion of short-term memory within each agent. Each ant remembers a small number of locations where it has successfully dropped an item. And so, when picking a new item this memory is consulted in order to bias the direction in which the ant will move. Thus, the ant tends to move towards the location it last dropped a similar item.

Lumer and Faieta [50] define picking up and dropping probabilities as follows:

$$
\begin{aligned}
& P_{p}\left(o_{i}\right)=\left(\frac{k_{1}}{k_{1}+f\left(o_{i}\right)}\right)^{2} \\
& P_{d}\left(o_{i}\right)=\left\{\begin{array}{c}
2 f\left(o_{i}\right) \text { when } \mathrm{f}\left(\mathrm{o}_{\mathrm{i}}\right)<k_{2} \\
1, \quad \text { when } \mathrm{f}\left(\mathrm{o}_{\mathrm{i}}\right) \geq \mathrm{k}_{2}
\end{array}\right. \\
& f\left(o_{i}\right)=\left\{\begin{array}{c}
\left.\frac{1}{s^{2}} \sum_{o_{j} \in \operatorname{Neigh}(s \times \mathrm{s})(\mathrm{r})\left[1-\frac{\mathrm{d}\left(\mathrm{o}_{\mathrm{i}}, o_{j}\right)}{\alpha}\right]}\right] \text { when } \mathrm{f}>0 \\
0,
\end{array}\right.
\end{aligned}
$$

where:

- $\mathrm{f}(\mathrm{oi})$ is a measure of the average similarity of data object oi with the other data object $\mathrm{oj}$ present in the neighborhood of oi

- $\quad \mathrm{d}(\mathrm{oi}, \mathrm{oj})$ is the dissimilarity between pair of objects (oi, oj)

- $\quad \alpha$ is a factor that defines the scale for dissimilarity

- $\mathrm{k}_{1}$ and $\mathrm{k}_{2}$ are two constants that play a role similar to $\mathrm{k}_{1}$ and $\mathrm{k}_{2}$ in the BM.

Gutowitz [29] called the agents basic ants, which have:

1) a finite memory, which is a register of length ' $n$ ' that records the presence or absence of objects at the ant's previous ' $\mathrm{n}$ ' locations;

2) an object manipulation capacity;

3) a function that gives the probability to manipulate an object proportionally;

4) to the values in memory and a random variable; and

5) the capability to execute Brownian motion

Although the basic ants have only local perceptual capabilities, they are able to promote global order. The mechanism underlying this phenomenon was carefully investigated by Gutowitz [29]. He proposed the complexity 
seeking ants, which are variants of the basic ants proposed by Deneubourg et al. [16]. The complexity-seeking ants are allowed to see local complexity and tend to perform action in regions of highest local complexity.

Gutowitz [29] would improve on this model by giving the ants, the capacity to sense the complexity (or entropy) of their vicinity. The entropy level of the work area was determined by the presence or absence of objects, so that a place completely empty or completely full would have the lowest entropy, and a checkered pattern would have the highest. The level of entropy of the surrounding would affect the propensity of the ants to take an action. In this way, in areas with low entropy the ants would not try to pick or drop anything. These complexity-seeking ants were thus able to avoid actions that did not contribute to the clustering process, performing their task more efficiently.

Gutowitz [29] has suggested to use of spatial entropy to track the dynamics of clustering. The spatial entropy Es at scale ' $s$ ' is defined by:

$$
E_{s}=\sum_{I \in S} P_{I} \log P_{I}
$$

where $P_{I}$ is the faction of all objects on the lattice that are found in s-patch I.

Oprisan et al. [62] proposed a variant of Denebourg et al.'s basic model [16], in which the influence of previously encountered objects is distributed by a time factor.

Bonabeau [9] also explored the influence of various weighting functions, especially those with short-term activation and long-term inhibition.

Among other social movements, Bonabeau et al. [8] have simulated the way, ants work collaboratively in the task of grouping dead bodies so, as to keep the nest clean. It can be observed that, with time the ants tend to cluster all dead bodies in a specific region of the environment, thus forming piles of corpses.

Monmarche [59] [60] combined the stochastic and exploratory principles of clustering ants with the deterministic and heuristic of the popular k-means algorithm in order to improve the convergence of the ant-based clustering algorithm. The proposed hybrid method is called AntClass and is based on the work of Lumer and Faieta [50]. The AntClass algorithm allows an ant to drop more than one object in the same cell, forming heaps of objects. Another important contribution of this algorithm is that it also makes use of hierarchical clustering, implemented by allowing ants to carry an entire heap of objects.

M. Dorigo et al. [24] presented in an Ant System and ACO which is a meta-heuristic approach based on the foraging behavior (a positive motivation) of real ants. It is based on the parameterized probabilistic model - the pheromone model.

N. Slimane, N. Monmarche and G. Venturini [70] proposed Ant-Class algorithm, which applies explorative and stochastic principles from the ACO meta-heuristic combined with deterministic and heuristic principles from k-means.

Ramos and Merelo [63] developed a novel strategy called ACLUSTER to tackle unsupervised clustering as well as data retrieval problems. This algorithm was employed for textual document clustering. The authors proposed the use of bio-inspired spatial transition probabilities, avoiding randomly moving agents, which may explore non-interesting regions. In this sense, ants do not move randomly like SACA, but according to transition probabilities that depend on the spatial distribution of pheromone across the environment. If a particular cluster disappears, the pheromone tends to evaporate from that location. This approach is interesting, because pheromone represents the swarm memory and all ants can benefit from it. In other words, the ants share a common memory.

V. Ramos, F. Muge and P. Pina [64] noticed that the SACA would generate a large quantity of small clusters. They modified the Ant-based clustering by changing the movement paradigm. While the previous works all relied on random moving ants, their ants would move according to a trail of pheromones left on clustering formations. This would reduce the exploration of empty areas, where the pheromone would eventually evaporate. This algorithm was applied to the classification of stone images. They studied the performance of the algorithm on continuous clustering [65] and showed that this improves clustering performance for the ant-based clustering system.

Handl and Meyer [33] applied ant-based clustering as the core of a visual document retrieval system for world wide web searches in which the basic goal is to classify on line documents by contents' similarity. The authors adopted an idea of short-term memory and employed ants with different speeds, also allowing them to jump. In addition, they introduced an adaptive scaling strategy, as well as some further modifications to achieve reliable results and to improve efficiency.

Labroche et al. [46] proposed a clustering algorithm, called ANTCLUST, based on a modeling of the chemical recognition system of ants. This system allows the construction of a colonial odor used for determining the ants' nest membership, such that ants can discriminate between nest mates and intruders.

Kanade and Hall [41] presented a hybridization of the ant systems with the classical Fuzzy C-Means algorithm (FCM) to determine the number of clusters in a given dataset automatically. In their fuzzy ant algorithm, at first the ant-based clustering are refined using the FCM algorithm.

Handl et al. [34] [35] proposed a scheme that enables an unbiased interpretation of the clustering solutions obtained by ant-based clustering algorithms. The authors argue that although many of the results obtained by ant algorithms look promising, there is a lack of knowledge about the actual performance of such algorithms. In order to overcome this limitation, they proposed a technique which is based on the application of agglomerative hierarchical clustering method to the positions of the data items on the grid. Taking into consideration the developed method, the results achieved by the ant-based clustering algorithm proposed by Handl and Meyer [33] are compared, using both synthetic and real datasets, with those obtained by two classical algorithms (k-means and agglomerative average link), showing that the ant-based algorithm performs well when compared with them. 
Abraham and Ramos [2] applied the ACLUSTER to discover the web usage patterns and thereafter a genetic programming approach to analyze the visitor trends.

N. Labroche, N. Monmarche and G. Venturini [48] presented Ant-Clust algorithm. In this algorithm, the ants proceed according to chemical properties and odors to recognize themselves as similar or not.

H. Azzag, N. Monmarche, M. Slimane and G. Venturini [4] presented a new clustering algorithm (AntTree) for unsupervised learning.

Handl et al. [36] changed the ants' movement policy so that the ants, after dropping an object, would teleport to the next isolated object, and pick it automatically.

C.F. Tsai, C.W. Tsai, H.C. Wu and T. Yang [74] proposed a novel clustering method called ant colony optimization with different favor algorithm (ACODF). In this algorithm, a direct adaptation of the ACO meta-heuristic for solving clustering problems. This algorithm performed better than the fast SOM k-means and genetic k-means algorithm.

Xiaohua $\mathrm{Xu}$ and Ling Chen Chen Y. [79] presented an artificial ants sleeping model (ASM) and an adaptive artificial ants clustering algorithm $\left(\mathrm{A}^{4} \mathrm{C}\right)$ to resolve the clustering problem in data mining by simulating the behaviors of gregarious ant colonies.

P.S. Shelokar, V.K. Jayaraman and B.D. Kulkarni [69] described an ant colony optimization methodology for optimally clustering ' $\mathrm{N}$ ' objects into ' $\mathrm{K}$ ' clusters. The algorithm employs distributed agents which mimic the way real ants find a shortest path from their nest to food source and back. This algorithm is implemented and tested on several simulated and real datasets. The performance of this algorithm is compared with other popular stochastic or heuristic methods viz. genetic algorithm, simulated annealing and tabu search. The results reveal that the proposed algorithm is effective in terms of quality.

Yun-Fei Chen, Chanadio Abdul Fattah, Yu-Shu Liu and Gangway Yan [86] presented a new heuristic density-based ant colony clustering algorithm (HDACC).

S. Schockaert, M. De Cock, C. Cornelis and E.E. Kerre [68] presented a clustering algorithm, inspired by the behavior of real ants simulated by means of fuzzy IF-THEN rules. In this algorithm, no additional information such as an initial partitioning of the data or the number of clusters, is needed. The proposed algorithm is efficient, robust and easy to use.

Diego Alejandro Ingaramo, Guillermo Leguizamon and Marcelo Errecalde [19] proposed a new version of the Ant-Tree algorithm called Adaptive Ant Tree (AAT), an approach inspired on the self-assembling behavior observed in some species of real ants. The proposed algorithm uses the original Ant-Tree algorithm as the first stage. Then, a re-assignation of ants to different clusters and combination of those clusters is carried out to give a better clustering model. The main features of AAT is given by its capacity of disconnecting ants from the tree under construction without increasing significantly the running time.

Vegard Hartmann [38] tried a different approach to the ant clustering algorithm by using evolution to train both the system's disparity function and move policy. Each ant would have a neural network which would take the objects of its vicinity as input, and return the move action, and the pick up or drop action, as outputs. By changing the evolutionary system fitness function, he was able to train the ants to create annular clusters, one cluster would be encircling another.

A. Vizine, L.N. de Castro, E.R. Hruschka and R.R. Gudwin [76] proposed an Adaptive Ant-Clustering Algorithm ( $\left.\mathrm{A}^{2} \mathrm{CA}\right)$. They have made a series of improvements to Lumer and Faieta's system [50]. $\mathrm{A}^{2} \mathrm{CA}$ is more robust in terms of the number of clusters found and tends to converge into good solution while the clustering process evolves. To achieve these goals, they proposed three main modifications in the SACA: 1) a cooling schedule for the parameter that controls the probability of ants picking up objects from the grid; $\quad 2$ ) a progressive vision field that allows ants to 'see' over a wider area; and 3) the use of pheromone function added to the grid as a way to promote reinforcement for the dropping of objects at more dense regions of the grid. These modifications favor an adaptive clustering process, in the sense that the proposed algorithm tends to converge to stable clusters.

Vizine et al. [77] presented a new algorithm called ACA which comes with a cooling scheme for picking probabilities. The local error function remains unchanged. This algorithm improves convergence.

J. Handl, J. Knowles and M. Dorigo [37] described an improved version of the heuristic, called Adaptive Time Dependent Transporter Ants (ATTA), incorporating adaptive heterogeneous ants, a time-dependent transporting activity, and a method that transforms the spatial embedding produced by the algorithm into an explicit partitioning. ATTA is then subjected to the most rigorous experimental evaluation of an ant-based clustering and sorting algorithm undertaken to date. They compared its performance with standard techniques for clustering and topographic mapping using a set of analytical evaluation functions and a range of synthetic and real data collections. Their results demonstrate the ability of ant-based clustering and sorting to automatically identify the number of clusters inherent in a data collection, and to produce high quality solutions. They have also devised the versions of ATTA such as ATTA-C (ATTA for clustering) and ATTA-TM (ATTA for topographic mapping).

This work is followed by the work of Tan et al. [71] which removes the ant metaphor from the method and presents a deterministic version of ant-based clustering algorithm.

S. Chi and C.C. Yang [13] developed a new method which integrates ant colony SOM and k-means for clustering analysis. This algorithm improves the traditional methods by the robust and efficient methods of any ant colony optimization.

Lotfi Admane, Karima Benatchba, Mouloud Koudil, Lamri Siad and Said Maziz [49] introduced a method called AntPart for the resolution of exclusive unsupervised classification. This method is inspired by the behavior of a particular species of ants called Pachycondyla apicalis. The performances of this method are compared with other methods such as AntClass, AntTree and AntClust.

Yan Yang and Mohamed S. Kamel [82] developed a multi-ant colonies approach for clustering data that consists of some parallel and independent ant colonies and a queen 
ant agent. Each ant colony process takes different types of ants moving speed and different versions of the probability conversion function to generate various clustering results with an ant-based clustering algorithm. These results are sent to the queen ant agent and combined by a hyper-graph model to calculate a new similarity matrix. The new similarity matrix is returned back to each ant colony process to re-cluster the data using the new information. Experimental evaluation shows that the average performance of the aggregated multi-ant colonies algorithms outperforms that of the single ant-based clustering algorithm and the popular k-means algorithm. The result also shows that the lowest outliers strategy for selecting the current data set has the best performance quality.

Salima Ouadfel and Mohamed Batouche [67] have presented an artificial ant clustering algorithm (AntClust), a new ant-based algorithm for image segmentation that uses the self-organizing and autonomous brood sorting behavior observed in real ants.

B. Gillner [28] investigated the performance of ACLUSTER [63] and ATTA [37], under the measures and datasets proposed by Handl et al. [37]. Based on performance results of both algorithms gathered from numerous runs, the results indicate weaknesses in the design of ACLUSTER while ATTA is well capable of clustering tasks. ATTA is able to detect the correct number of clusters in the data.

Xiaofang Huang, Yixian Yang and Xinxin Niu [80] described an improved version, called chaotic ant clustering algorithm (CACAS), adopting an important strategy of using chaotic perturbation to improve individual quality and utilized chaos perturbation to avoid the search being trapped in local optimum. The performance of the proposed ant algorithm is compared with the k-means approach and ant-based clustering by evaluation functions and topographic mapping using a set of analytical data. The experimental results demonstrate the proposed method is a robust and viable.

Jianhua $\mathrm{Qu}$ and Xiyu Liu [40] presented a quick and effective ant clustering (QAC) algorithm.

Warangkhana Ngenkaew, Satoshi Ono and Shigeru Nakayama [78] proposed a method of using two kinds of deposited pheromones at each grid, Trailing Pheromone and Foraging Pheromone in ant-based clustering algorithm.

Ying Wang, Ren-Wang Li, Bin Li, Peng-Ju Zhang and Yao-Hui Li [84] proposed an advanced clustering method called ant colony ISODATA algorithm (ACIA) in real time computer simulation.

Zhang Tao, Lv Xiaodong and Zhang Zaixu developed an improved clustering algorithm based on ant colony approach. This algorithm is applied in aggregation analysis. The experimental results reveal that the proposed ant algorithm is effective, efficient and quick convergence.

Urszula Boryczka [75] presented a new ant clustering algorithm called ACA for data clustering in a knowledge discovery context. In this algorithm, he employed a modified version of the short-term memory introduced by Lumer and Faieta [50] in order to improve the convergence. The proposed algorithm is evaluated in a number of well-known benchmark data sets.

In this ACA, the following threshold formulae are used for picking and dropping decisions :

$$
\begin{aligned}
& P_{\text {pit }}^{*}(i)= \begin{cases}1, & \text { if } f^{*}(i)>1 \\
\frac{1}{f^{*}(i)^{2}}, & \text { else }\end{cases} \\
& P_{\text {dop }}^{*} \text { (i) }= \begin{cases}1, & \text { if } f^{*}(i) \geq 1 \\
\frac{1}{f^{*}(i)^{4}}, & \text { else }\end{cases}
\end{aligned}
$$

where $f^{*}(i)$ is a modified version of Lumer and Faieta's neighborhood function:

$$
f^{*}(i)=\left\{\begin{array}{c}
\frac{1}{\sigma^{2}} \sum_{j}\left[1-\frac{d(i, j)}{\alpha}\right], \text { if } f^{*}>0 \\
\text { and }\left(1-\frac{d(i, j)}{\alpha}\right)>0 \\
0, \text { otherwise }
\end{array}\right.
$$

C. Fernandes, A.M. Mora, J.J. Merelo, V. Ramos and J.L.J. Laredo [26] have introduced a new method for clustering and data classification, based on an hybridization of Ant Algorithms and Kohonen Self-Organizing Maps. It has been demonstrated that KohonAnts (or KANTS ) model is useful for clustering and classification tasks, yielding very good results in both kind of problems.

Lutz Herrmann and Alfred Ultsch [52] presented a new work that shows how the ant-based clustering algorithm from Lumer and Faieta [50] is related to Self-Organizing Maps [45]. They omitted the mechanism of picking and dropping ants for a formal analysis of the underlying formulae. They analyzed the popular technique of Lumer/Faieta. The Lumer/Faieta approach is strongly related to Kohonen's Self-Organizing Batch Map. In their work, a unifying basis is derived in order to assess strengths and weaknesses of both techniques. The behavior of popular derivatives of LF such as ACLUSTER, ACA and ATTA is also explained.

Ashish Ghosh, Anindya Halder, Megha Kothari and Susmita Ghosh [3] presented a new algorithm for clustering data sets based on the property of aggregation pheromone found in ants, called APC. An ant is placed at each location of a data point, and the ants are allowed to move in the search space to find points with pheromone density. The movement of an ant is governed by the amount of pheromone deposited at different points of the search space. More the deposited pheromone, more is the aggregation of ants. This leads to the formation of homogenous groups of data.

Zahra Sadeghi, Mohammad Teshnehlab and Mir Mohsen Pedram [88] presented a new strategy for clustering using artificial ants in which groups of ants try to do clustering by inserting and removing operations. Clustering is done using groups of ants which are as many as the number of clusters. The goal of each group is to collect members of one cluster. All data objects and ants are spread randomly on the grid. Each ant contains a load list which is initialized with a random object at first. Ants search the grid and try to collect similar data to their load. The load list of ants of each group constructs each cluster. That is, the proposed method uses ' $\mathrm{k}$ ' ants for finding ' $\mathrm{k}$ ' clusters and they collect data objects in their load list, so there is no need to the retrieving process. This algorithm outperforms k-means algorithm and LF model 
of ant clustering. The two new probability functions of picking and dropping are given below:

$$
\begin{aligned}
& P_{\text {irvest }}(i)=\left\{\begin{array}{cl}
\left(\frac{f(i)}{k_{\text {ix }}+f(i)}\right)^{2}, & \text { if } f(i)<k_{\text {ixs }} \\
1, & \text { if } f(i) \geq k_{\text {ixs }}
\end{array}\right. \\
& P_{\text {renore }}(i)=\left\{\begin{array}{cl}
0, & \text { if } f(i) \geq k_{\text {ren }} \\
\left(\frac{k_{\text {ren }}}{k_{\text {ren }}+f(i)}\right)^{2}, & \text { if } f(i)<k_{\text {ren }}
\end{array}\right.
\end{aligned}
$$

where Kins and Krem are the inserting and removing constants respectively.

Qin Chen and Jinping Mo [61] proposed an improved ant clustering algorithm based on k-means, which optimizes the rules of ant clustering algorithm.

Zhao Weili [90] proposed an improved entropy-based ant clustering (IEAC) algorithm. The information entropy to model behaviors of agent is applied. The better quality clusters can be obtained by using the entropy function.

Fei Wang, Dexian Zhang and $\mathrm{Na}$ Bao [25] proposed a method of document clustering algorithm based on Ant Colony Algorithm (ACO) and Fuzzy C-means clustering (FCM). This algorithm makes use of Ant Colony Algorithm for finding local extremum. After obtaining good initial clusters, Fuzzy C-means clustering is applied.

This is not the exhaustive list of all techniques, other approaches can also be found in the literature. A number of modifications have been introduced to the basic ant-based clustering scheme that improve the quality of the clustering, convergence, stability and other key features.

\section{APPLICATIONS}

Ant-based clustering algorithms have been used in a large variety of applications. They are applied for web usage mining. Web usage mining attempts to discover useful knowledge from the secondary data obtained from the interactions of the users with the web. A. Abraham and V. Ramos [2] proposed an ant-based clustering algorithm to discover Web usage patterns (data clusters) and a linear genetic programming approach to analyze the visitor trends. The ant colony inspired methods can also be applied in many stages of the Electrocardiogram Interpretation Process [56] [57] [58]. Initially, the Deneubourg and LF algorithms have become well-known models that have been used in applications like data mining [60] [79] and graph-partitioning [47]. Several papers have reported successful applications of ant-based clustering algorithms such as Data retrieval and textual document clustering [63], Classification of stone images [64], Web usage mining [2] [31], Solvency prediction [87], Network traffic analysis [72], Intrusion detection [66], Long-term electrocardiogram processing [55], Textural defect detection [11], Knowledge discovery in DNA chip analysis data [54], Distributed databases [14], Biomedical data processing [53], Image segmentation [67] [81] [83], Human skin analysis and generating portal site [31], Image retrieval [30], Content-based image retrieval [73], Data clustering [75], Clustering and classification [26], Computer forensics [17] and Gene expression data analysis [85].

\section{CONClusion AND FurTher RESEARCh Directions}

Ant-based clustering algorithms are an appropriate alternative to traditional clustering algorithms. The algorithm has a number of features that make it an interesting study of cluster analysis. It has the ability of automatically discovering the number of clusters. It linearly scales against the dimensionality of data. The nature of the algorithm makes it fairly robust to the effects of outliers within the data. Research on ant-based clustering algorithms is still an on-going field of research. In this paper, we address a brief survey of ant-based clustering algorithms and an overview of some of its applications. There are a number of directions in which research on ant-based clustering can be continued. We summarize and conclude the survey with listing some important future works and research trends for ant-based clustering algorithms: a comparative study of ant clustering performance with respect to other clustering algorithms; applying ant clustering algorithms to real-world applications; effects on performance of user-defined parameters; a hierarchical analysis of the input data by varying some of the user-defined parameters; sensitivity analysis of various user-defined parameters of ant clustering algorithms; to determine optimal values of parameters other than pick and drop policies; developing new probabilistic rules for picking and dropping objects; study the effect based on reasonably good validity index function to judge the fitness of several possible partitioning of the data of ant-based clustering schemes and validating mathematically; study the possibility of dynamic clustering using ant clustering with data mining applications; applying ant clustering algorithms for multi-objective optimization problems; study of transformation of ant clustering algorithms into supervised algorithms; developing new theoretical results of behavior of ant clustering algorithms and study of hierarchical ant-based clustering algorithms; to analyze the working principles that ant-based clustering shares with other clustering methods; hybridization of ant-clustering algorithm with alternative clustering methods.

\section{REFERENCES}

[1] A. Abraham, He Guo and Hongbo Liu, "Swarm Intelligence: Foundations, Perspectives and Applications", Swarm Intelligence in Data Mining, A. Abraham, C. Crosan, V. Ramos (Eds.), Studies in Computational Intelligence (series), Springer, Germany, 2006.

[2] A. Abraham and V. Ramos, "Web usage mining using artificial ant colony clustering and linear genetic programming", Proc. Congress on Evolutionary Computation (IEEE Press), Australia, 2003, pp. 1384-1391.

[3] Ashish Ghosh, Anindya Halder, Megha Kothari and Susmita Ghosh, "Aggregation pheromone density based data clustering", Information Sciences, Vol. 178, Issue 13, 1 July 2008, pp. 2816-2831.

[4] H. Azzag, N. Monmarche, M. Slimane and G. Venturini, “AntTree: a new model for clustering with artificial ants", Evolutionary Computation, CEC'03, Vol. 4, 2003, 2642-2647.

[5] P. Berkhin, "Survey clustering Data Mining Techniques", Technical Report, Accrue Software, San Jose, California, 2002.

[6] W. Bin and S. Zhongzhi, "A clustering algorithm based on swarm intelligence", Proc. of the Int. Conf. on Info-tech. and Info-net, Beijing, China, 2001, pp. 58-66. 
[7] Marco Dorigo and Thomas Stutzle, "Ant Colony Optimization", Prentice-Hall of India Private Ltd., New Delhi, 2006.

[8] E. Bonabeau, M. Dorigo and G. Theraulaz, "Swarm Intelligence: from natural to artificial systems", Oxford University Press, Inc., New York, NY, 1999.

[9] E. Bonabeau, "From Classical Models of Morphogenesis to Agent-Based Models of Pattern Formation", Artificial Life, 1997, 3:191-209.

[10] S. Camazine, J.-L. Deneubourg, N.R. Franks, J.Sneydd, G. Theraulaz and E. Bonabeau, "Self-Organization in Biological Systems", Princeton University Press, 2001

[11] Chao Zou, Li Xiao and Bingwen Wang, "Textural defect detect using a revised ant colony clustering algorithm", MIPPR 2007.

[12] L. Chretien, "Organization Spatiale du Materiel Provenant de L'excavation du nid chez Messor Barbarus et des Cadavres d'ouvrieres chez Lasius niger \{Hymennopterae: Formicidae)", Ph.d. thesis, Universite Libre dr Bruxelles, 1996

[13] S. Chi and C.C. Yang, "Integration of Ant Colony SOM and k-means for Clustering Analysis", Knowledge Based Intelligent Information and Engineering Systems, LNCS, Springer, Vol. 4251, 2006, pp. 1-8.

[14] R. Chandrasekar and T.Srinivasan, "An Improved Probabilistic Ant based Clustering for Distributed Databases", IJCAI 2007, Proceedings of the 20th International Joint Conference on Artificial Intelligence, Hyderabad, India, Jan. 6-12, 2007, pp. 2701-2706.

[15] B. Duran and P. Odell, "Cluster Analysis: A Survey", New York: Springer-Verlag, 1974.

[16] J.-L. Deneubourg, S. Gross, N. Franks, A. Sendova-Franks, C. Detrain and L. Chretien, " The dynamics of collective sorting: Robot-like ants and ant-like robots", In Proceedings of the First International Conference on Simulation of Adaptive Behavior: From Animals to Animats, Cambridge, MA, MIT Press, 1991, pp. 356-363.

[17] Deguang Wang and Lili Hao, "Application of Ant Colony Clustering in Computer Forensics", Proceedings of the 2009 Second International Conference on Information and Computing Science, Vol. 2, 2009, pp. $87-90$.

[18] G. DI Caro and M. Dorigo, "Extending AntNet for Best-effort Quality-of-Services Routing", Ant Workshop on Ant Colony Optimization, http://iridia.ulb.ac.be/ants98/ants98.html, 1998, pp. 15-16.

[19] Diego Alejandro Ingaramo, Guillermo Leguizamon and Marcelo Errecalde, "Adaptive clustering with artificial ants", Journal of Computer Science and Technology, Science Press, Vol. 5, No. 4, 2005

[20] M. Dorigo, V. Maniezzo and A. Colorni, "Ant system: optimization by a colony of cooperating agents", IEEE Transaction on Systems, Man and Cybernetics- Part B, 26(1), 1996, pp. 29-41.

[21] M. Dorigo, "Optimization, learning and natural algorithms", Ph.D. Thesis, Dipartimento di Elettronica, Politecnico di Milano, Italy, 1992.

[22] Marco Dorigo and Thomas Stutzle, "The Ant Colony Optimizaion Metaheuristic: Algorithms, Applications, and Advances", In: F. Glover and G. Kochenberger (Eds.), Handbook of Metaheuristics, Kluwer Academic Publishers.

[23] M. Dorigo , E. Bonabeau and G. Theraulaz, "Ant algorithms and stigmergy", Future Generation Computer Systems, 16(8), 2000, pp. 851-871.

[24] M. Dorigo, G. Di Caro and L.M. Gambarella, "Ant Algorithms for Discrete Optimization", Artificial Life, 5(3), 1999:137-172.

[25] Fei Wang, Dexian Zhang and Na Bao, "Fuzzy Document Clustering Based on Ant Colony Algorithm", Proceedings of the 6th International Symposium on Neural Networks: Advances in Neural Networks - Part II, Lecture Notes in Computer Science, Vol. 5552, 2009, pp. 709-716.

[26] C. Fernandes, A.M. Mora, J.J. Merelo, V. Ramos and J.L.J. Laredo, "KohonAnts: A Self-Organizing Ant Algorithm for Clustering and Pattern Classification", http://arxiv.org/abs/0803.2695v1, 2008.

[27] N.R. Franks and A.B. Sendova-Franks, "Brood sorting by ants: distributing the workload over the work surface", Behav. Ecol. Sociobiol., 1992, 30:109-123.

[28] B. Gillner, "A comparative study of ant clustering algorithms", M.Sc. Thesis, University of Maastricht Department of Mathematics, Netherlands, Oct. 22, 2007

[29] H. Gutowitz, "Complexity-seeking ants", In Proc. of the Third European Conference on Artificial Life, 1993.

[30] Haifang Li, Xia Wen and Hui Jin, "The Clustering Algorithm Research of Image Emotional Characteristics Based on Ant Colony", Proceedings of the 2008 International Symposium on Intelligent Information Technology Application Workshops, 2008, pp. 455-458.

[31] Hanene Azzag, Gilles Venturini, Antoine Oliver and Christiane Guinot, "A hierarchical ant based clustering algorithm and its use in three real-world applications", European Journal of Operational Research, Vol. 179, Issue 3, 16 June 2007, pp. 906-922.

[32] J. Han and M. Kamber. "Data Mining: Concepts and Techniques", Morgan Kaufmann, San Francisco, 2001.

[33] J. Handl, J and B. Meyer, "Improved Ant-Based Clustering and Sorting in a Document Retrieval Interface", Proceedings of the 7th International Conference on Parallel Problem Solving from Nature, LNCS 2439, 2002.

[34] J. Handl, J. Knowles and M. Dorigo, "On the performance of ant-based clusterin", Proc. of the Third International Conference on Hybrid Intelligent Systems Frontiers in Artificial Intelligence and Appliations, IOS Press, Vol. 104, 2003, pp. 204-213.

[35] J. Handl, J. Knowles and M. Dorigo, "Ant-based clustering: a comparative study of its relative performance with respect to k-means, average link and 1 d-som", Technical Report TR/IRIDIA/2003 24, IRIDIA, Universite Libre de Bruxelles, http://www.handl.julia.de., 2003.

[36] J. Handl, J. Knowles and M. Dorigo, "Strategies for the increased robustness of ant-based clustering, Lecture Notes in Computer Science, Vol. 2977, 2004, pp. 90-104.

[37] J. Handl, J. Knowles and M. Dorigo, "Ant-Based Clustering and Topographic Mapping”, Artificial Life, MIT Press, Vol. 12, No. 1, 2006, pp.35-61.

[38] V. Hartmann, "Evolving agents swarms for clustering and sorting", In Genetic Evolutionary Computation Conference, GECCO, ACM Press, Vol. 1, 2005, pp. 217-224.

[39] A.K. Jain, M.N. Murty and P.J. Flynn, "Data Clustering: a review", ACM Computing Surveys, Vol. 31, 1999, pp. 264-323.

[40] Jianhua Qu and Xiyu Liu, "A Quick Ant Clustering Algorithm", Proceedings of the Fourth International Conference on Fuzzy Systems and Knowledge Discovery, Vol. 01, 2007, pp. 722-725.

[41] P. Kanade and L.O. Hall, "Fuzzy ants as a clustering concepts", Proc. of the 22nd International Conference of the North American Fuzzy Information Processing Society (NAFIPS), 2003, pp. 227-232.

[42] J. Kennedy and R. Eberhart, "Swarm Intelligence", Morgan Kaufmann Publishers, Inc., San Francisco, CA, 2001.

[43] J. Kennedy and R. Eberhart, "Particle Swarm Optimization", IEEE International Conf. on Neural Networks, Piscataway, NJ, Vol. 4, 1995, pp. 1942-1948.

[44] S.B. Kotsiantis and P.E. Pintelas, "Recent Advances in Clustering: A Brief Survey".

[45] T. Kohonen, "Self-Organizing Maps", Springer series in Information Sciences, Vol. 30, Springer, Berlin, Heidelberg, New York, 1995, 1997, 2001.

[46] N. Labroche, N. Monmarche and G. Venturini, “ A new clustering algorithm based on the chemical recognition system of ants", Proc. of the 15th European Conference on Artificial Intelligence, IOS Press, France, 2002.

[47] A. E. Langham and P.W. Grant, "Using Competing Ant Colonies to Solve k-way Partitioning Problems with Foraging and Raiding Strategies", In: D. Floreano et al. (Eds.), Advances in Artificial Life, Lecture Notes in Computer Science, Springer, 1999, pp. 621-625.

[48] N. Labroche, N. Monmarche and G. Venturini, "AntClust: Ant Clustering and Web Usage Mining", In Genetic and Evolutionary Computation Conference, Chicago, 2003, pp. 25-36.

[49] Lotfi Admane, Karima Benatchba, Mouloud Koudil, Lamri Siad and Said Maziz, "AntPart: an algorithm for the unsupervised classification problem using ants", Applied Mathematics and Computation, Vol. 180, Issue 1, 2006, pp. 16-28.

[50] E.D. Lumer and B. Faieta, "Diversity and adaptation in populations of clustering ants", Cambridge: MIT Press, In D. Cliff, P. Husbands, J.-A. Meyer, \& S.W. Wilson (Eds.), From animals to animats: Proceedings of the Third International Conference on Simulation of Adaptive Behavior, 1994, pp. 501-508

[51] E. Lumer and B. Faieta, "Exploratory database analysis via self-organization", 1995.

[52] Lutz Herrmann and Alfred Ultsch, "Explaining Ant-Based Clustering on the basis of Self-Organizing Maps", ESANN2008 Proceedings, European Symposium on Artificial Neural Networks - Advances in Computational Intelligence and Learning, Bruges (Belgium), April 2008, pp. 23-25.

[53] Miroslav Bursa and Lenka Lhotska, "Ant Colony Inspired Clustering in Biomedical Data Processing", In 3 rd European Symposium on Nature-inspired Smart Information Systems [CD-ROM], Aachen: NiSIS, 2007

[54] Minsoo Lee, Yun-mi Kim, Yearn Jeong Kim, Yoon-kyung Lee and Hyejung Yoon, "An Ant-based Clustering System for Knowledge 
Discovery in DNA Chip Analysis Data", Proceedings of World Academy of Science, Engineering and Technology, 23, Aug. 2007.

[55] Miroslav Bursa and Lenka Lhotska, "Modified Ant Colony Clustering Method in Long-term Electrocardiogram Processing", Engineering in Medicine and Biology Society 2007 (EMBS 2007), 29th Annual International Conference of the IEEE, 2007, pp. 3249-3252.

[56] Miroslav Bursa, Michal Huptych, Lenka Lhotska, "The Use of Nature Inspired Methods in Electrocardiogram Analysis", In Intelligent Data Engineering and Automated Learning - Proceedings of IDEAL 2006. Berlin: Springer, International Special Topics Conference on Information Technology in Biomedicine [CD-ROM], Piscataway: IEEE, 2006, pp. 1390-1398.

[57] Miroslav Bursa and Lenka Lhotska, "The Use of Ant Colony Inspired Methods in Electrocardiogram Interpretation, an Overview", In NiSIS2006 - The 2nd European Symposium on Nature-inspired Smart Information Systems [CD-ROM], Aachen: NiSIS, 2006.

[58] Miroslav Bursa and Lenka Lhotska, "Nature Inspired Concepts in the Electrocardiogram Interpretation Process", Computers in Cardiology, Vol. 35, 2008, pp. 241-244.

[59] N. Monmarche, M. Slimane and G. Venturini, "On improving clustering in numerical databases with artificial ants", Advances in Artificial Life, 1999, pp. 626-635.

[60] N. Monmarche, "On Data Clustering with Artificial Ants", In: Freitas AA, (ed.), Data Mining with Evolutionary Algorithms: Research Directions - Papers from the AAAI Workshop, AAAI Press, pp. 23-26.

[61] Qin Chen and Jinping Mo, "Optimizing the Ant Clustering Model Based on k-means Algorithm", Proceeding of the 2009 WRI World Congress on Computer Science and Information Engineering, Vol. 03, 2009, pp. 699-702.

[62] S.A. Oprisan, V. Holban and B. Moldoveanu, "Functional Self-Organization Performing Wide-Sense Stochastic Processes”, Phys Lett., 1996, A 216:303-306.

[63] V. Ramos and J.J. Merelo, "Self-Organized Stigmergic Document Maps: Environment as a Mechanism for Context Learning", In E. Alba, F. Herrera, J.J. Merelo et al. Eds., AEB'2002, First Spanish Conference on Evolutionary and Bio-inspired Algorithms, Spain, 2002, pp. 284-293

[64] V. Ramos, F. Muge and P.Pina, "Self-Organized Data and Image Retrieval as a Consequence of Inter-DynamicSynergistic Relationships in Artificial Ant Colonies", In J. Ruiz-del-Solar, A. Abrahan and M. Koppen Eds., Soft-Computing Systems - Design, Management and Applications, Frontiers in Artificial Intelligence and Applications: IOS Press, Amsterdam, v. 87, 2002, pp. 500-509.

[65] V. Ramos and Ajith Abraham, "Swarms on continuous data", In CEC'03 Congress on Evolutionary Computation, IEEE Press, 2003, pp 1370-1375

[66] V. Ramos and A. Abraham, "Antids: Self-Organized Ant-Based Clustering Model For Intrusion Detection System", Proceedings of the Fourth IEEE International Workshop, WSTST'05, Muroran, Japan, 2005, pp. 977-986.

[67] Salima Ouadfel and Mohamed Batouche, "An Efficient Ant Algorithm for Swarm-based Image Clustering", Journal of Computer Science, Science Publications, 3(3), 2007, pp. 162-167.

[68] S. Schockaert, M. De Cock, C. Cornelis and E.E. Kerre, "Efficient Clustering with Fuzzy Ants", Applied Computational Intelligence, World Scientific Publishing Company, 2004.

[69] P.S. Shelokar, V.K. Jayaraman and B.D. Kulkarni, "An ant colony approach for clustering", Analytica Chimica Acta, Vol. 509, Issue 2, 3 May 2004, pp. 187-195.

[70] N. Slimane, N. Monmarche and G. Venturini, "Antclass: discovery of clusters in numeric data by an hybridization of an ant colony with k-means algorithm", Rapport interne 213, Laboratoire d' Informa-tique de l' Universite de Tours, E3i Tours, 1999.

[71] S.C. Tan, K.M. Ting and S.W.T., " Reproducing the Results of Ant-based Clustering without using Ants", CEC 2006, IEEE Congress on Evolutionary Computation, 2006, pp. 1760-1767.

[72] Teemu Ekola, Mikko Laurikkala, Timo Lehto and Hannu Koivisto, "Network Traffic Analysis Using Clustering Ants", World Automation Congress (WAC 2004), Sevilla, Spain, June 2004.

[73] Toomas Piatrik and Ebroul Izquierdo, "An Application of Ant Colony Optimization to Image Clustering", In Proc. K-Space Jamboree Workshop, 2008.

[74] C.F. Tsai, C.W. Tsai, H.C. Wu and T. Yang, "Acodf: a novel data clustering approach for data mining in large databases", Journal of System Software, 2004, 73(1), pp. 133-145.

[75] Urszula Boryczka, "Ant Clustering Algorithm", Intelligent Information Systems, Kluwer Academic Publishers, 2008, pp. 377-386.
[76] A. Vizine, L.N. de Castro, E.R. Hruschka and R.R. Gudwin, "Towards improving clustering ants: An adaptive clustering algorithm", Informatica Journal, Vol. 29, 2005.

[77] A. L. Vizine, L. N. de Castro, R. R. Gudwin, "Text Document Classification Using Swarm Intelligence", In: International Conference on Integration of Knowledge Intensive Multi-Agent Systems, 2005, pp. 134-139.

[78] Warangkhana Ngenkaew, Satoshi Ono and Shigeru Nakayama, "Multiple pheromone deposition in ant-based clustering as an ant foraging concept", Proceeding of the third conference on IASTED International Conference on Advances in Computer Science and Technology, 2007, pp. 432-436.

[79] Xiaohua Xu and Ling Chen Chen Y., "A4C: an adaptive artificial ants clustering algorithm", Proceedings of the 2004 IEEE Symposium on Computational Intelligence in Bioinformatics and Computational Biology, 2004, CIBCB'04, 2004, pp. 268-275.

[80] Xiaofang Huang, Yixian Yang and Xinxin Niu, "Towards improving Ant-based clustering - An Chaotic Ant Clustering Algorithm", Proceedings of the 2007 International Conference on Computation Intelligence and Security Workshops, 2007, pp. 421-424.

[81] Xiaochun Yang, Weidong Zhao, Yufie Chen and Xin Fang, "Image segmentation with a fuzzy clustering algorithm based on Ant-Tree", Signal Processing, Vol. 8, Issue 10, 2008, pp.2435-2462.

[82] Yan Yang and Mohamed S. Kamel, "An aggregated clustering approach using multi-ant colonies algorithms", Pattern Recognition, Vol. 39, Issue 7, July 2006, pp. 1278-1289.

[83] Yanfang Han and Pengfei Shi, "An improved ant colony algorithm for fuzzy clustering in image segmentation", Neurocomputing, Vol. 70, Issues 4-6, Jan. 2007, pp. 665-671.

[84] Ying Wang, Ren-Wang Li, Bin Li, Peng-Ju Zhang and Yao-Hui Li, "Research on an Ant Colony ISODATA Algorithm for Cluster Analysis in Real Time Computer Simulation", Proceedings of the Second Workshop on Digital Media and its Application in Museum \& Heritage, 2007, pp. 223-229.

[85] Yulan He and Siu Cheung Hui, "Exploring ant-based algorithms for gene expression data analysis", Artificial Intelligence in Medicine, In Press, Corrected Proof, Available on line 18 April 2009.

[86] Yun-Fei Chen, Chanadio Abdul Fattah, Yu-Shu Liu and Gangway Yan, "HDACC: A Heuristic Density-Based Ant Colony Clustering Algorithm", Proceedings of the Intelligent Agent Technology, IEEE/WIC/ACM International Conference, 2004, pp. 397-400.

[87] Yue Xi, Qiang Han and Anthony Brabazon, "An Ant-Clustering Model for Solvency Prediction", Proc. of the International Conference on Artificial Intelligence, IC-AI'04, Vol. 2, 2004, pp. 687-690.

[88] Zahra Sadeghi, Mohammad Teshnehlab and Mir Mohsen Pedram, "K-Ants Clustering - A New Strategy Based on Ant Clustering", ICS 2008, Tamkang, Vol. 2, 12 Feb. 2009, pp. 329-334.

[89] Zhang Tao, Lv Xiaodong and Zhang Zaixu, "An Improved Clustering Algorithm Based on Ant Colony Approach", Proceedings of the 2007 International Conference on Computational Intelligence and Security Workshops, 2007, pp. 437-440.

[90] Zhao Weili, "An Improved Entropy-Based Ant Clustering Algorithm", Proceedings of the 2008 WASE International Conference on Information Engineering, Vol 2, 2009, pp. 41-44.

First A. Mohamed Jafar O.A. He obtained his M.Phil. in Computer Science from Bharathidasan University Tiruchirappalli in 1998. Currently he is working as a Associate Professor at Department of Computer Science, Jamal Mohamed College (Autonomous), Tiruchirappalli, Tamil Nadu, India. $\mathrm{He}$ is doing research in Computer Science in the Department of Computer Science, A.V.V.M. Sri Pushpam College (Autonomous), Poodi, Thanjavur, Tamil Nadu, India under Bharathidasan University, Tiruchirappalli. His areas of interests include Data Mining, Clustering, Swarm Intelligence and Evolutionary Algorithms.

Second B. Sivakumar R. He obtained his Ph.D. in Computer Science from Bharathidasan University, Tiruchirappalli. Currently he is working as a Associate Professor and Head of the Department at Department of Computer Science, A.V.V.M. Sri Pushpam College (Autonomous), Poodi, Thanjavur, Tamil Nadu, India. His areas of interests include Human-Computer Interaction, Neural Networks, Artificial Intelligence, Swarm Intelligence and Evolutionary Algorithms. 